\title{
Facial Privacy Preservation using FGSM and Universal Perturbation attacks
}

This paper was downloaded from TechRxiv (https://www.techrxiv.org).

\section{LICENSE}

CC BY 4.0

SUBMISSION DATE / POSTED DATE

$28-12-2021 / 11-01-2022$

CITATION

J, Nishchal (2022): Facial Privacy Preservation using FGSM and Universal Perturbation attacks. TechRxiv. Preprint. https://doi.org/10.36227/techrxiv.17696489.v1

DOI

10.36227/techrxiv.17696489.v1 


\title{
Facial Privacy Preservation using FGSM and Universal Perturbation attacks
}

\author{
Nishchal Jagadeesha \\ $R V$ College of Engineering \\ nishchalj.cs18@rvce.edu.in
}

\begin{abstract}
Research done in Facial Privacy so far has entrenched the scope of gleaning soft-biometric attributes such as gender, race and age from a human's face image. Many techniques have been proposed to ensure user privacy, such as visible distortions to the images, manipulation of the original image with new face attributes, face swapping etc. Though these techniques achieve the goal of user privacy by fooling face recognition models, they don't help the user to upload original images without visible distortions or manipulation. The objective of this work is to implement techniques to ensure the privacy of user's sensitive or personal data in face images by creating minimum pixel level distortions using white-box and black-box perturbation algorithms to fool AI models while maintaining the integrity of the image, so as to appear the same to a human eye.
\end{abstract}

Keywords- Data Privacy, Facial Privacy, Black Box Attack, White Box attack, Fast Gradient Sign Method(FGSM), Universal Perturbation, Facial Recognition, Privacy attributes, CNN, Adversarial Machine Learning, DeepFool algorithm.

\section{INTRODUCTION}

The swift growth of the Information Age has surpassed and neglected the legal infrastructure for protecting an individual's privacy. While the existing laws are limited to protect the privacy of citizens in most countries around the world with respect to "analog" data. Inclusion of digital data is fairly difficult due to the fact that it can be easily replicated, shared and even stolen. Even in cases where laws do exist, for example, the European Union General Data Protection Regulation, it is difficult to enforce when data can be transferred to other legal jurisdictions. Image data is particularly concerning because of its invasive applications and the lack of protection from these practices in existing laws[2]. Due to the absence of strict policies to safeguard the digital privacy rights of a person, time has come where the users themselves have to take up precautions and necessary measures to protect their privacy as other protection measures are not in place. A common feature to be focused on in several online social media platforms is the photo tagging. Most open source public applications that offer media storage services like Google, Facebook and Flickr, make use of facial recognition tools to tag different individuals in the photos. Though, this is an attraction to some consumers, it is a great risk of privacy for many others. This paper discusses the possible approaches to protect user privacy by impersonating the face which is the most sensitive and unique feature of an individual.

Many techniques have been proposed to ensure user privacy, such as visible distortions to the images, manipulation of the original image with new face attributes or face swapping, addition of special features like hats, glasses, a beard, a different smile, etc. Though these techniques achieve the goal of user privacy by fooling face recognition models, they don't help the user to upload original images without distortions or manipulation. Techniques that provide pixel level changes, without distorting the original image achieve better results with the aid of Adversarial Machine Learning algorithms that look at how neural networks can be fooled by feeding them with deceptive inputs. It also ensures that the originality of the image will be maintained.

This paper proposes two models, a black box model that involves generation of a universal perturbation for the image using a DeepFool algorithm and a white box model which involves an FGSM attack on the image for generating a noise mask for the image. The results of all these algorithms are tested against face recognition models to ensure proper misclassification. 


\section{Literature Survey}

By definition, facial identification is a classification task. Mere classification only permits a fixed number of output classes in the network, which is obviously impractical for facial recognition because the network would have to be re-trained every time a new person was to be added to the database. The solution is to transition from a classification task to a more regression-like task with networks that generate meaningful representations of faces in the form of numerical vectors. There are various black-box approaches proposed like the GenAttack-with gradient free optimization which uses a lesser number of queries to form the adversarial image compared to zeroth order optimization (ZOO). Here, genetic algorithms were used for synthesising adversarial examples. Work has been done on MNIST, CIFAR-10, ImageNet datasets, but nothing has been done specifically for human faces. In particular for faces, work has been done by generating non-invasive noise masks to apply to facial images for a newly introduced user, yielding adversarial examples and preventing the formation of identifiable clusters in the embedding space. The algorithms proposed are executed in a white-box environment, which may not always be accessible and also it doesn't include application of feature extractor in facial recognition models.[7]

Semi-adversarial networks are another way of persevering attributes of faces. Generally there is a possibility of deducing soft-biometric attributes such as age, gender and race from an individual's face image with high accuracy. This raises privacy concerns and to tackle this scenario, a technique was developed for transfering soft biometric privacy to face images via an image perturbation methodology which also gives the user the choice to obfuscate specific attributes from the input face image. Though the idea was commendable, the results were not quite satisfactory. The modified images from the proposed model had some artifacts. As a result, a human observer will be able to distinguish between perturbed face images and non-modified one.[8] With an effort to address privacy issues systematically, balance usability, and enhance privacy in a natural and measurable manner a framework- AnonymousNet was proposed. The stack involves 4 stages namely facial attribute estimation, privacy-metric-oriented face obfuscation, directed natural image synthesis, and adversarial perturbation. But the limitation here was evaluation of perturbation performance among different deep neural network-based detectors qualitatively and quantitatively, and was ignored, due limitations in space and computational resources.[9] Another creative approach was proposed which let users add minor pixel-level changes ("cloaks") to their own photos which did not affect the visual anatomy of the image. When used to train facial recognition models, these "cloaked" images produce functional models that consistently cause normal images of the user to be misidentified. But it was accurate only for the Microsoft Azure API.[10]

Doubly Permuted Homomorphic Encryption is another way of achieving data privacy. Here, the framework is designed to aggregate multiple classifiers updated locally using private data and to ensure that no private information about the data is exposed during and after its learning procedure. They utilize a homomorphic cryptosystem that can aggregate the local classifiers while they are encrypted and thus kept secret. By using homomorphically encrypted locally-updated classifiers, the aggregator can average them while ensuring that the classifiers never expose private information about the trained. But, it is primarily focused exclusively on the learning of linear classifiers (like SVM). A promising direction for the future work is learning much higher-dimensional models like sparse convolutional neural networks.[11] GAN models for learning private and fair representations were proposed, which uses adversarial learning to allow a data holder to learn universal representations of a given dataset that decouple a set of sensitive attributes from the rest of the dataset. It involves modifying the training data that decouple a set of sensitive attributes from the non-sensitive ones. But, this model hasn't been tested against a large dataset. The size of the dataset may affect the convergence speed of the decorrelation schemes.[12]

Not much work has been dedicated completely for personal data theft, but various approaches have been proposed to achieve data privacy, but each one of these approaches have had some limitations, either the dataset or accuracy etc. Hence, observing the gaps in the works already done gives a fair idea as to what improvements can be done.[13] 


\section{Objectives and Proposed Methodology}

Adversarial machine learning can be used to implement techniques that are cyber attacks by nature, which can be categorised based on the resources available to the attacker:

1. Create pixel level distortions to images. When these images whose pixels are subjected to minor changes are used to train facial recognition models, they produce weights that regularly result in misclassification of normal images of the user, thus imparting privacy. This model will be a white box implementation.

2. Generate non-invasive noise masks to apply to facial images for a newly introduced user and hence prevent the formation of identifiable clusters in the embedding space. Such protection is offered through a system that alters images in a manner that is indistinguishable to the human eye without having prior knowledge about the facial recognition model architecture, and yet large enough to cause misclassifications. This model will be a black box implementation.[3]

\section{A. Fast Gradient Sign Method (FGSM) - White Box Attack}

Any adversarial attack is termed as a white-box if the weights, loss functions and other hyper-parameters of target Neural network model are known to the attacker in prior, that will be used for performing the attack.

The fast gradient sign method operates by using the gradients of the neural network to create an adversarial example. For an input image, it calculates the gradients of the loss with respect to the input image which will produce a perturbation noise matrix. Using this matrix, a new adversarial image will be created which gets misclassified by the model. This can be summarised using the following expression as shown in eqn 1 [15]:

$$
a d v_{x}=\mathcal{X}+e^{*} \operatorname{sign}\left(\operatorname{del}_{x} J(\text { theta,x,y)) }\right.
$$

where

$a d v_{x}:$ Adversarial image.

$\mathcal{X}$ : Original input image.

y : Original input label.

e : Multiplier to secure small perturbations.

theta : Model parameters.

$\mathrm{J} \quad$ : Loss function
The system architecture explains the workflow of the White box model attack. For a given dataset, the faces present in the images will be extracted using the MTCNN model. This data is used to train a facial classifier.

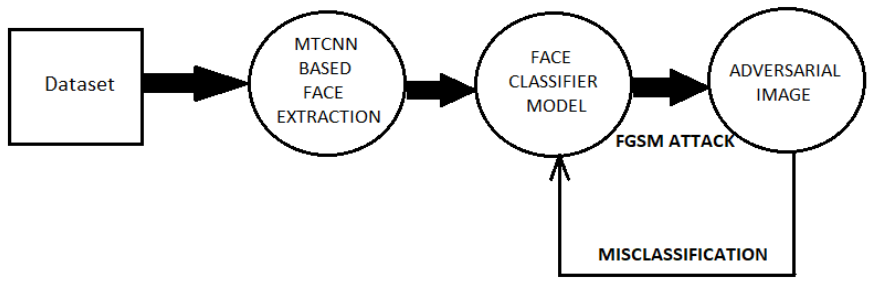

Fig 1: System architecture of White box model

The trained classifier's Hyperparameters are known to the attacker. Using the loss gradient of the predicted class and the input given to the classifier, FGSM generates a perturbation, which when added to the Image, adversarial properties are generated. The workflow of the process is shown in fig 1 .

\section{B. Black Box Attack - Universal Perturbations}

The adversarial attacks are called the black-box attacks in case no information about the target $\mathrm{CNN}$ is available.[4][5] Universal perturbations proposes a method for estimating a single perturbation matrix which when added to any image from a particular dataset, the image transforms into an adversary. This perturbation is termed as universal, because it represents a fixed image-agnostic perturbation that misclassifies an entire dataset of images belonging to $\mu$. The focus here is on the case where the distribution $\mu$ represents the set of natural images, hence containing a huge amount of variability. The goal is to find perturbation $(\boldsymbol{v})$ that satisfies the following two constraints as shown in eqn 2 and eqn 3 [16]:

$$
|v| \leq \xi
$$

$$
P(\check{\mathrm{K}}(x+v) !=\check{\mathrm{K}}(x)) \geq 1-\delta
$$

The parameter $\xi$ controls the magnitude of the perturbation vector $\boldsymbol{v}$, and $\delta$ quantifies the desired fooling rate for all images sampled from the distribution. Let $X=\{x 1, \ldots, x m\}$ be a set of images sampled from the distribution $\mu$. The proposed algorithm calculates a universal perturbation $\boldsymbol{v}$, such that $|\mathrm{v}| \leq \xi$, while fooling 
all/most data points in $\mathrm{X}$. The algorithm proceeds in a loop over the images in $\mathrm{X}$ and gently updates the universal perturbation. At each iteration, the minimal perturbation $\Delta \boldsymbol{v}$ i transmits the current perturbed point, $\mathrm{xi}+\boldsymbol{v}$, classifier's decision boundary is computed, and summed up to the current sample of the universal perturbation using the update rule as shown below.

$$
\begin{aligned}
& \Delta \boldsymbol{v} i \leftarrow \operatorname{argmin}|r| \text { s.t. } \check{\mathrm{K}}(\mathrm{xi}+\boldsymbol{v}+\mathrm{r}) !=\check{\mathrm{K}}(\mathrm{xi}) \\
& \mathrm{Pp}, \xi(\boldsymbol{v})=\operatorname{argmin}\left|\boldsymbol{v}-\boldsymbol{v}^{\prime}\right| \text { subject to }\left|\boldsymbol{v}^{\prime}\right| \leq \xi
\end{aligned}
$$

The quality of universal perturbation can be improved with several iterations on the data set X. The algorithm is terminated when the empirical "fooling rate" on the perturbed data set $\mathrm{X} \boldsymbol{v}:=\{\mathrm{x} 1+\boldsymbol{v}, \ldots, \mathrm{xm}+\boldsymbol{v}\}$ exceeds the target threshold $1-\delta$. That is, we stop the algorithm whenever eqn 5 [16] is met.

$$
\operatorname{Err}(\mathrm{X} \boldsymbol{v})=1 / \mathrm{m} * \Sigma(\check{\mathrm{K}}(\mathrm{xi}+\boldsymbol{v}) !=\check{\mathrm{K}}(\mathrm{xi})) \geq 1-\delta
$$

The system architecture explains the workflow of the Black box model attack. For a given dataset, an initial perturbation is randomly generated and based on its misclassifying rate with respect to any face classifier available, a universal perturbation is estimated.

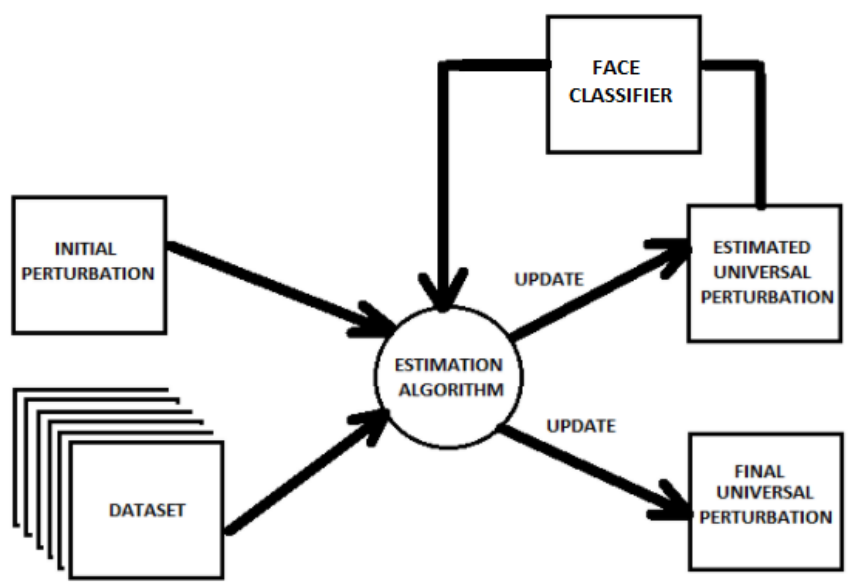

Fig 2: System architecture of Black box model

After several iterations updates, a final perturbation is obtained which misclassifies majority of the images in the dataset when added to them. The workflow of the process is shown in fig 2 .

\section{Experimental Analysis}

\section{A. Fast Gradient Sign Method}

Following is the detailed structural representation of the workflow of White box model. It consists of a MTCNN model for face extraction followed by a custom face classifier and the FGSM attack.

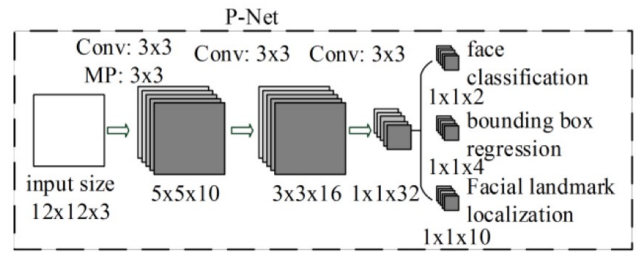

Fig 3: MTCNN(P-NET)

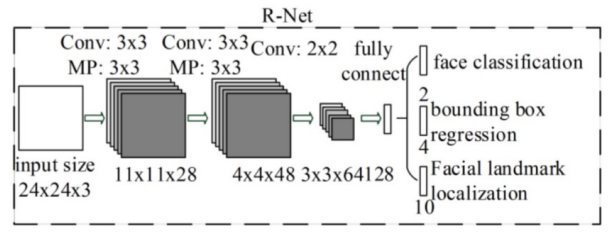

Fig 4: MTCNN(R-NET)

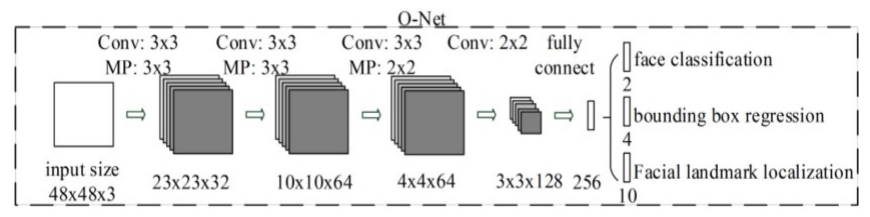

\begin{tabular}{|c|c|c|}
\hline Layer (type) & Output Shape & Param \# \\
\hline conv2d (Conv2D) & (None, $160,160,8$ ) & 224 \\
\hline max_pooling2d (MaxPooling2D) & (None, $80,80,8$ ) & 0 \\
\hline conv2d_1 (Conv2D) & (None, $80,80,16$ ) & 1168 \\
\hline max_pooling2d_1 (MaxPooling2 & (None, 26, 26, 16) & 0 \\
\hline conv2d_2 (Conv2D) & (None, 26, 26, 32) & 4640 \\
\hline max_pooling2d_2 (MaxPooling2 & (None, $8,8,32$ ) & 0 \\
\hline flatten (Flatten) & (None, 2048) & 0 \\
\hline dense (Dense) & (None, 512) & 1049088 \\
\hline dense_1 (Dense) & (None, 55) & 28215 \\
\hline $\begin{array}{l}=========================== \\
\text { Total params: } 1,083,335 \\
\text { Trainable params: } 1,083,335 \\
\text { Non-trainable params: } 0\end{array}$ & & \\
\hline
\end{tabular}

Fig 5: MTCNN(O-NET)

Model: "sequential"

Fig 6: Custom face classifier 


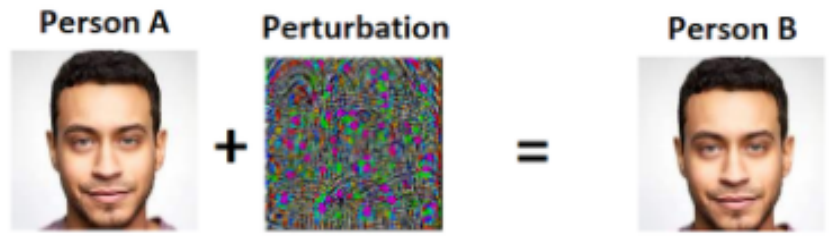

Fig 7: FGSM Attack

Multi-task Cascaded Convolutional Networks (MTCNN) is used for Face Detection and Facial Landmark Alignment, i.e. face extraction. This first stage is a fully convolutional network (FCN) as shown in fig 3. The Proposal Network is responsible for producing candidate windows and the bounding box regression vectors. All candidates from the P-Net are fed into the Refine Network as shown in fig 4. The R-Net helps in further reduction of candidates, also calibrates it with bounding box regression vectors and employs NMS (non-maximum suppression) to combine overlapping candidates. The final outcome of R-Net is to classify the input as a face or not, and a 4 element vector containing the bounding box pixel coordinates of the faces present in the image. O-NET further improvises the results by identifying landmark features of the face such as eyes, nose and mouth to describe the face in more detail.

A custom neural network is built for face classification as shown in fig 6. Three convolutional 2D layers are used with filter size 8,16 and 32 respectively. After each Conv2D layer, max pooling is done. Finally the layers are flattened and two dense layers are used. Activation function used is ReLU with Adam optimiser. The loss considered here is categorical cross entropy.

The core step of the attack is performing FGSM Attack with the gradients of the loss w.r.t the input image to create an adversarial image that maximises the loss and eventually causes misclassification. A perturbation matrix is calculated by finding out how much each pixel of the image contributes to loss value. Using the chain rule to find required gradients and loss of each input pixel of image makes this process very fast and easy. Fig 7 shows the expected result after an FGSM attack is done on the facial recognition model.

\section{B. Universal Perturbation}

The structure of the facial classifier remains the same as explained in the FGSM attack. The blacbox attack here is done on the dataset directly instead of on the model. Model's architecture is not known prior to the attack.

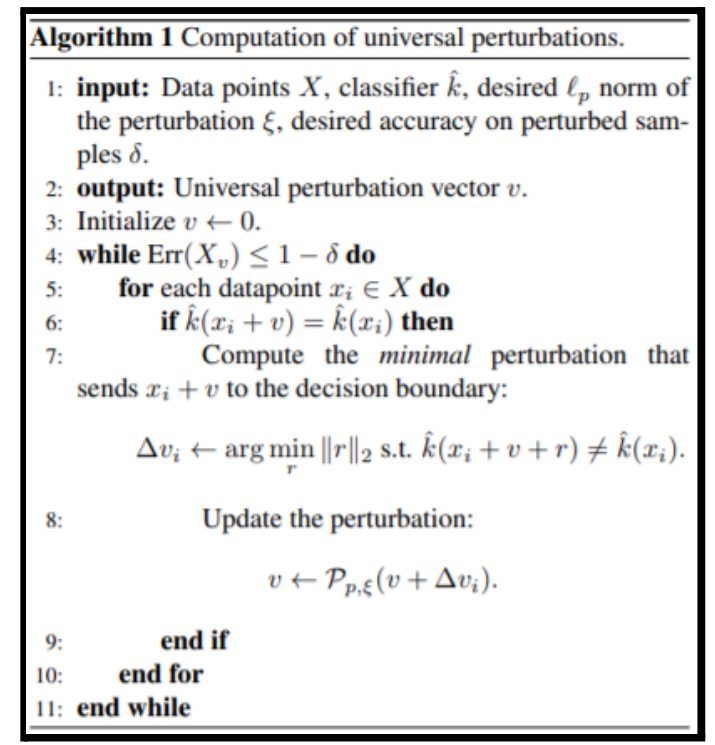

Fig 8: Universal Perturbation

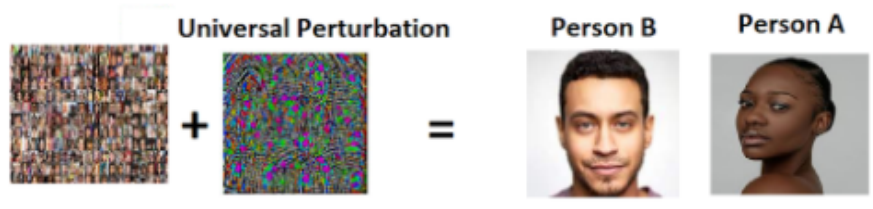

Fig 9: Universal Perturbation

Universal perturbations proposes a method for estimating a single perturbation matrix which when added to any image from a particular dataset, the image transforms into an adversary. The focus here is on the case where the distribution $\mu$ represents the set of natural images, hence containing a huge amount of variability. In that context, possible examination of the existence of small universal perturbations that misclassify most images is done. Fig 8 shows the algorithm used to derive the perturbation matrix. Fig 9 shows the possible result after the attack is done on the facial recognition model.

The training was done on GPU: 1xTesla K80, compute 3.7, having 2496 CUDA cores, 12GB GDDR5 VRAM. 


\section{Experimental Results and Testing}

In order to evaluate the performance of the approaches, accuracy is formulated as, the ratio of number of images in the dataset that are misclassified by the facial recognition model to the total size of the dataset. This accuracy roughly gives an idea about the level of privacy that can be obtained when this approach is being used. The class that has the greatest probability is considered as the actual class. So, more the difference in probabilities in each of the classes of faces, gives a better and clear prediction of the attacked class. The Georgia Tech face database (128MB) dataset used in the training process consists of images of 50 people taken in two or three sessions between 06/01/99 and 11/15/99 at the Center for Signal and Image Processing at Georgia Institute of Technology. All the images in the database are represented by 15 color JPEG images with a cluttered background taken at resolution 640x480 pixels. The mean dimension of the faces in these images is $150 \times 150$ pixels. The faces are subjected to many orientations, tilt, expressions, lighting conditions and scaling to increase variability in the dataset. The ground truth bounding box is created by manually labeling each face in the images.

The accuracy reached 0.99 for the training set with a loss of 0.0367 . Below are the training and testing details of the model.

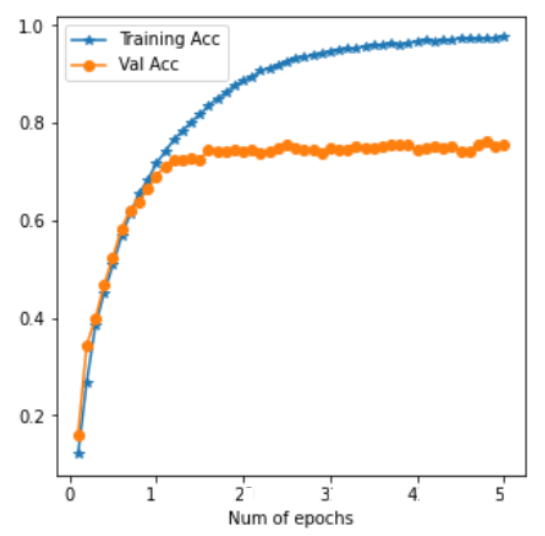

Fig 10: Training of Face Recognition Model

The FGSM approach, by far, produces the best results as the architecture of the facial recognition model is known and used to perform the attack. Thus, the facial recognition model whose architecture uses neural networks can easily be fooled with an accuracy of almost $100 \%$ can be achieved. Greater the difference in probabilities of prediction that a particular image belongs to a particular class, better is the performance of the approach. Hence, it shows that image is properly misclassified by fooling the facial recognition model.

The Universal Perturbation matrix generated by this approach when applied to the faces of the dataset successfully misclassifies upto $64 \%$ of the faces with a single perturbation matrix with 49 iterations. The number of iterations run is proportional to the degree of misclassification. The results can be seen in the tables.

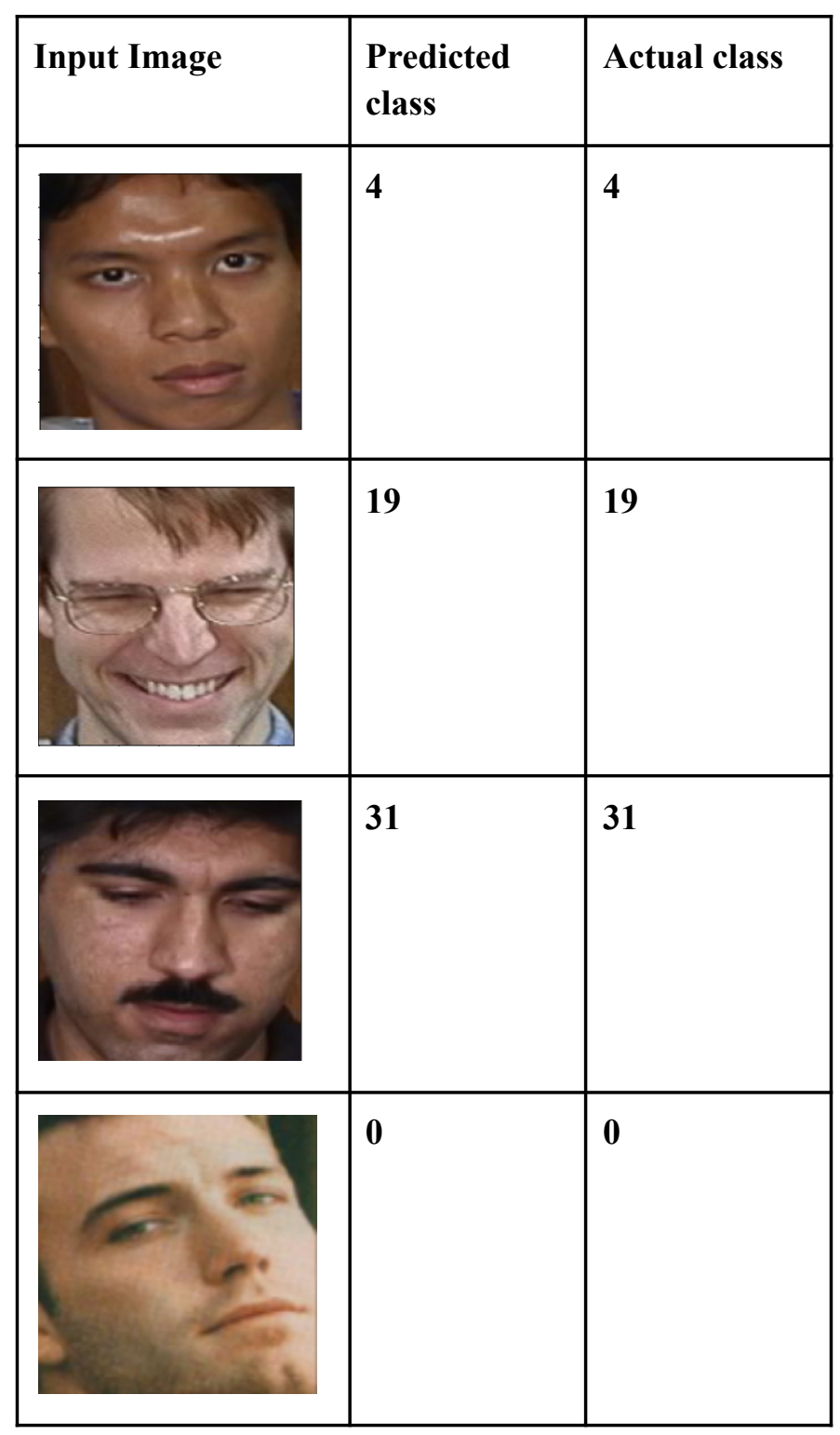

Table 1: Face Recognition Model Results 

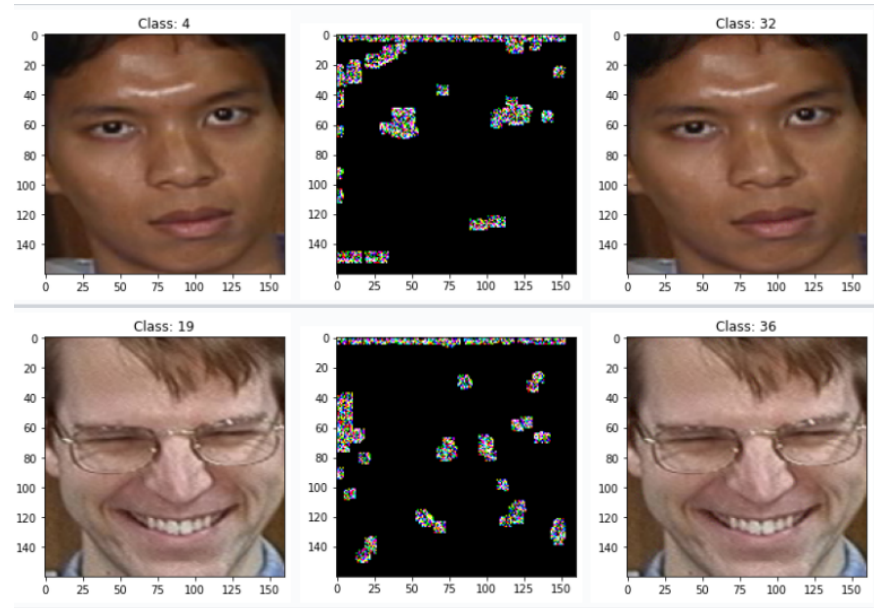

Class: 31
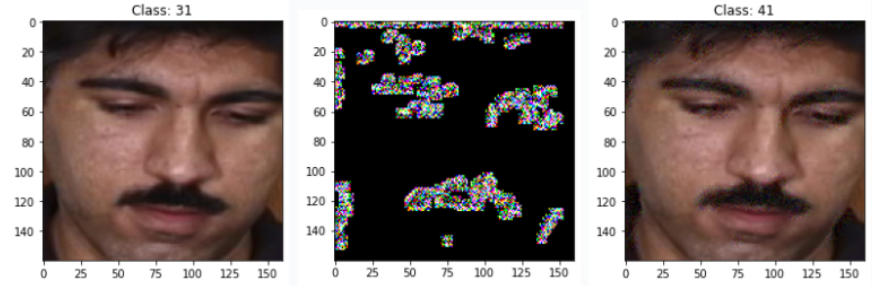

Table 2: Results of FGSM Attacks - (Original Image, Perturbation obtained, Output Image) labelled with class output of the facial recognition model.

White box attack was carried out on the custom trained classifier. Images from the test dataset were taken, attacked and tested on the classifier model. The system testing of the white box model is shown in table 2 .

Black box attack was carried out on the custom trained classifier. Images from the dataset were taken, attacked and tested on the classifier model. The system testing of the Black box model is shown in table 7 .

\section{Limitations And Future Scope}

The black box model doesn't achieve good results for a larger dataset. The white box model doesn't work if the architecture of the facial recognition model is not known clearly. The white box model can be improved so that it can work on facial recognition models built based on feature extractions. The black box model works well for smaller datasets, but it's misclassifying rate decreases as dataset size increases as it fails to accommodate for all the images in one perturbation. A mechanism to handle larger datasets has to be researched upon.

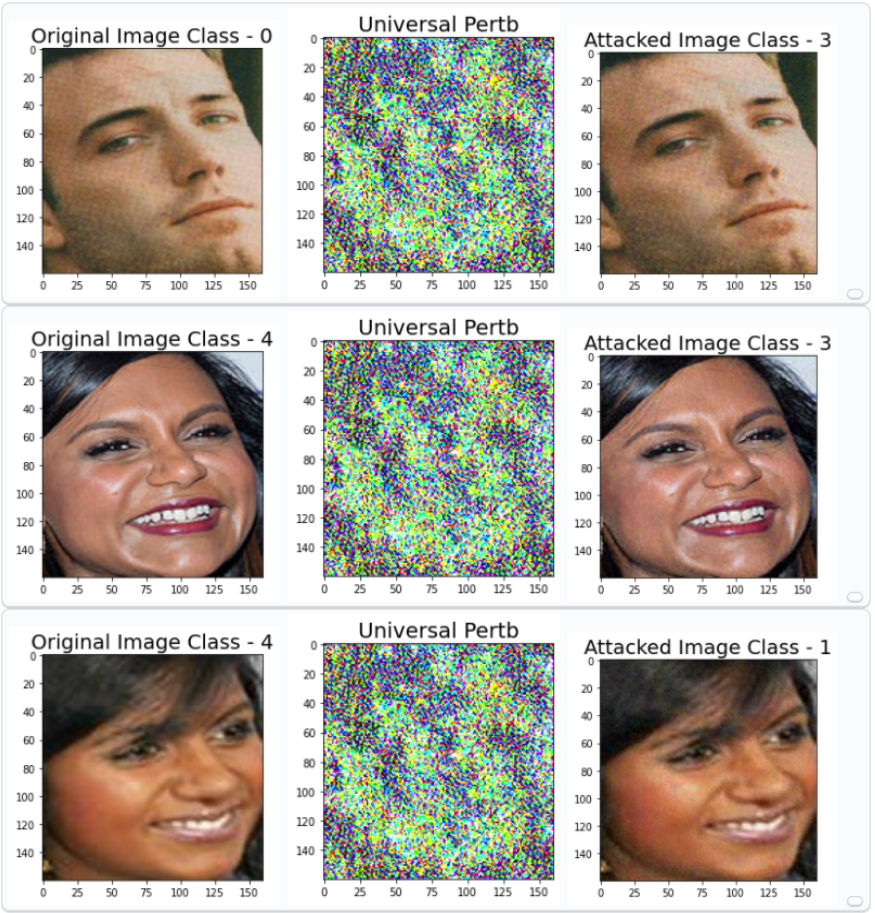

Table 3: Results of Universal Perturbation Attacks (Original Image, common Universal Perturbation, Output Image) labelled with class output of the facial recognition model.

VII. Conclusion

Deep learning algorithms are used in a variety of fields including data analytics, successfully solving a variety of problems such as image classification, natural language processing, and prediction of consumer behavior. The triumph of these algorithms pivots on the accessibility of large image datasets, which most probably contains sensitive data about the subject that might facilitate learning models to inherit societal biases leading to unintended algorithmic discrimination on legally protected groups such as race or gender [17][18]. This has led to growth of research on transforming sensitive data to fair and private representations.

The techniques in this paper provide a new approach to handle privacy issues. The perturbations generated through white box and black box approaches can fool the neural network and achieve user privacy. Though, more work has to be done in generalising these approaches to the benchmarked facial recognition systems, the work in this paper provides a starting point for the same! 
[1] Y. Lin, H. Zhao, X. Ma, Y. Tu and M. Wang, "Adversarial Attacks in Modulation Recognition With Convolutional Neural Networks," in IEEE Transactions on Reliability, vol. 70, no. 1, pp. 389-401, March 2021, doi: 10.1109/TR.2020.3032744.

[2] Thomas Cilloni, Wei Wang, Charles Walter, Charles Fleming, University of Mississippi Oxford, USA and Xi'an Jiaotong-Liverpool University Suzhou, China, "Preventing Personal Data Theft in Images with Adversarial ML", https://arxiv.org/abs/2010.10242, May 2020.

[3] Xiaoyong Yuan, Pan He, Qile Zhu, Xiaolin Li, National Science Foundation Center for Big Learning, University of Florida,"Adversarial Examples: Attacks and Defenses for Deep Learning", https://arxiv.org/pdf/1712.07107, July 2018.

[4] Siddhant Bhambri, Sumanyu Muku, Avinash Tulasi, Arun Balaji Buduru," A Survey of Black-Box Adversarial Attacks on Computer Vision Models", https://arxiv.org/abs/1912.01667, December 2019.

[5] Huiying Li, Shawn Shan, Emily Wenger, Jiayun Zhang, Haitao Zheng, Ben Y. Zhao," Blacklight: Defending Black-Box Adversarial Attacks on Deep Neural Networks", https://arxiv.org/abs/2006.14042, June 2020.

[6] D. Jayaraman, F. Sha and K. Grauman, "Decorrelating Semantic Visual Attributes by Resisting the Urge to Share," 2014 IEEE Conference on Computer Vision and Pattern Recognition, 2014, pp. 1629-1636, doi: 10.1109/CVPR.2014.211.

[7] Moustafa Alzantot, Yash Sharma, Supriyo Chakraborty, Huan Zhang, Cho-Jui Hsieh, Mani Srivastava,"GenAttack:Practical Black-box Attacks with Gradient-Free Optimization", https://arxiv.org/abs/1805.11090v3, May 2018.

[8] Shawn Shan, Emily Wenger, Jiayun Zhang, Huiying Li, Haitao Zheng, and Ben Y. Zhao, University of Chicago, "Fawkes: Protecting Privacy against Unauthorized Deep Learning Models", 29th USENIX Security Symposium, January 2020.

[9] Tao Li, Lei Lin, Department of Computer Science Purdue University and Goergen Institute for Data Science University of Rochester, "AnonymousNet: Natural Face De-Identification with Measurable Privacy", https://arxiv.org/pdf/1904.12620, October 2019.

[10] Vahid Mirjalili, Sebastian Raschka, Arun Ross, Department of Computer Science and Engineering, Michigan State and University Department of Statistics, University of Wisconsin - Madison, " PrivacyNet: Semi-Adversarial Networks for Multi-attribute Face Privacy", https://arxiv.org/pdf/2001.00561, June 2020.

[11] Ryo Yonetani, The University of Tokyo, Japan, Vishnu Naresh Boddeti, Michigan State University USA,Kris M. Kitani, Carnegie Mellon University PA, USA, Yoichi Sato, The University of Tokyo, Japan," Privacy-Preserving Visual Learning Using Doubly Permuted Homomorphic Encryption", https://arxiv.org/pdf/1704.02203, July 2017.

[12] Chong Huang and Xiao Chen and Peter Kairouz and Lalitha Sankar and Ram Rajagopal, "Generative Adversarial Models for Learning Private and Fair Representations", ICLR Conference, New Orleans, Louisiana, United States, May 2019.

[13] H. Ismail Fawaz, G. Forestier, J. Weber, L. Idoumghar and P. Muller, "Adversarial Attacks on Deep Neural Networks for Time Series Classification," 2019 International Joint Conference on Neural Networks (IJCNN), 2019, pp. 1-8, doi: 10.1109/IJCNN.2019.8851936.

[14] N. Narodytska and S. Kasiviswanathan, "Simple Black-Box Adversarial Attacks on Deep Neural Networks," 2017 IEEE Conference on Computer Vision and Pattern Recognition Workshops (CVPRW), 2017, pp. 1310-1318, doi: 10.1109/CVPRW.2017.172.

[15] J. Xu, Z. Cai and W. Shen, "Using FGSM Targeted Attack to Improve the Transferability of Adversarial Example," 2019 IEEE 2nd International Conference on Electronics and Communication
Engineering (ICECE), 2019, pp. 20-25, doi: 10.1109/ICECE48499.2019.9058535.

[16] S. Moosavi-Dezfooli, A. Fawzi, O. Fawzi and P. Frossard, "Universal Adversarial Perturbations," 2017 IEEE Conference on Computer Vision and Pattern Recognition (CVPR), 2017, pp. 86-94, doi: 10.1109/CVPR.2017.17.

[17] Y. Zhou, M. Han, L. Liu, J. He and X. Gao, "The Adversarial Attacks Threats on Computer Vision: A Survey," 2019 IEEE 16th International Conference on Mobile Ad Hoc and Sensor Systems Workshops (MASSW), 2019, pp. 25-30, doi: 10.1109/MASSW.2019.00012.

[18] B. Xu, J. Zhu and D. Wang, "Adversarial Attacks for Object Detection," 2020 39th Chinese Control Conference (CCC), 2020, pp. 7281-7287, doi: 10.23919/CCC50068.2020.9188998.

[19] Tao Li, Lei Lin, Department of Computer Science Purdue University and Goergen Institute for Data Science University of Rochester, "AnonymousNet: Natural Face De-Identification with Measurable Privacy", https://arxiv.org/pdf/1904.12620, October 2019.

[20] Shawn Shan, Emily Wenger, Jiayun Zhang, Huiying Li, Haitao Zheng, and Ben Y. Zhao, University of Chicago, "Fawkes: Protecting Privacy against Unauthorized Deep Learning Models", 29th USENIX Security Symposium, January 2020. 